\title{
International consortium unveils numerous new cancer genetic markers
}

$\mathrm{T}$ he largest genome-wide study focused on oncology has discovered 74 new genetic variants linked to breast, ovarian and prostate cancers.

The recent coordinated release of 13 papers by the Collaborative Oncological Gene-environment

Study (COGS) details the massive effort that involved 160 research centres and uncovered genetic markers associated with high-risk or aggressive disease. While the newly identified genetic variations, known as single nucleotide polymorphisms (SNPs), partly explain the heritability of cancer, there are many disease-causing SNPs that likely remain undiscovered.

SNPs are most commonly discovered in the DNA between genes, although they can also occur within a

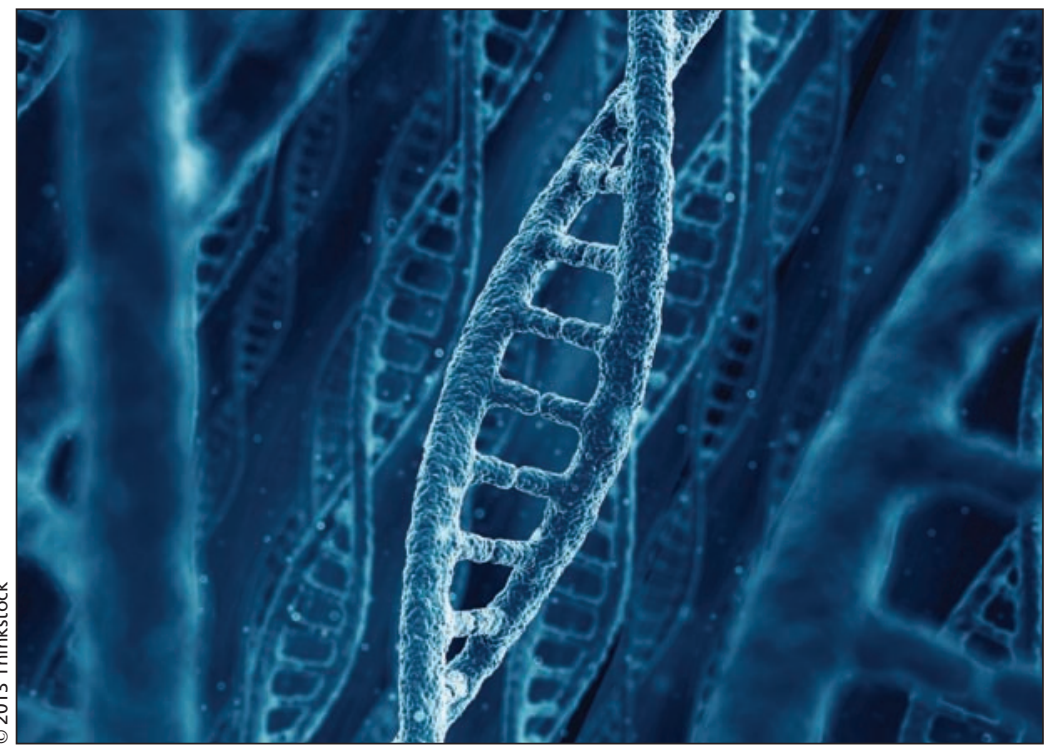

The 13 new studies uncovered genetic markers known as single nucleotide polymorphisms associated with high-risk or aggressive disease. a more accurate risk prediction. BRCA genes are members of a class of tumoursuppressing genes which, when mutated, are associated with hereditary breast cancer representing $5 \%$ to $10 \%$ of all breast cancers in women.

Identifying the high-risk SNPs asso- an aggressive preventive treatment such as mastectomy, versus those with lower risk, who could be routinely evaluated for any changes.

The COGS study also found breast, ovarian and prostate cancer - all hormone-driven - may have a common cause. The study found 18 loci, or points on chromosomes, associated with more than one cancer.

"We already had a clue that ovarian cancer and estrogen receptornegative breast cancer look similar at the tumour level," says Couch. "We found that several loci are common between the two diseases, which was very interesting and suggests that they have a common etiology."

Because each SNP only has a minute effect on the risk of developing cancer, the study needed large gene or in a regulatory region near a gene. Researchers use them because they can act as biomarkers to help locate genes associated with cancer and other diseases. In the body, they can more directly affect a gene's function.

Researchers estimate that the proportion of familial risk genetics explained is now $30 \%$ for prostate cancer, $28 \%$ for breast cancer and $4 \%$ for ovarian cancer. Finding other causative rare mutations will involve "a mix of statistics and molecular biology," says Fergus Couch, an investigator at the Mayo Clinic in Rochester, Minnesota, and author of two COGS publications.

Couch believes carriers of breast cancer (BRCA) gene mutations could benefit most from these studies, because the findings could help geneticists make ciated with these genes means women may be able to change their clinical strategy. That's because women with the 10 SNPs that Couch and his colleagues have identified as modifying BRCA1 would have a higher risk of developing breast cancer than those without these SNPs.

"These women have a 65 to $90 \%$ lifetime risk of breast cancer, versus the average woman who has a $12 \%$ risk," says Couch. "For BRCA1 and BRCA2 carriers, this is a tremendous impact."

Women in the top 5\% of risk for breast cancer based on their SNPs, for example, have a 2.4-fold increased chance of developing the disease compared with women who, based on their SNPs, are at lower risk. Women at that increased risk might then benefit from numbers of subjects - in this case, more than 250000 - to confirm the most important variants.

"The number of cases and controls [in COGS] is unprecedented," says Raju Kucherlapati, a professor in Harvard Medical School's genetics department who was not involved in the study. The resulting effect sizes - that is, how much the SNPs contribute to cancer risk - are also significant and larger than in past studies, he added.

"We clearly see now that SNPs, put together, really do serve as an important risk factor," says Couch. "This has the promise of changing the way we do personalized risk prediction."Amanda Alvarez, Berkeley, Calif.

CMAJ 2013. DOI:10.1503/cmaj.109-4467 